\title{
ERRATUM
}

\section{Molecular Surveillance of Cryptosporidium spp. in Raw Wastewater in Milwaukee: Implications for Understanding Outbreak Occurrence and Transmission Dynamics}

\author{
Ling Zhou, Ajaib Singh, Jianlin Jiang, and Lihua Xiao \\ Division of Parasitic Diseases, Centers for Disease Control and Prevention, Atlanta, Georgia 30341, and \\ City of Milwaukee Public Health Laboratories, Milwaukee, Wisconsin 53202
}

Volume 41, no. 11, p. 5254-5257, 2003. Page 5254, column 2, lines 1 to 4 from bottom: “primer sets 5'-ATAGTCTCGCTGT ATTC-3' and 5'-GCAGAGGAACCAGCATC-3' in the primary PCR and 5'-TCCGCTGTATTCTCAGCC-3' and 5'-GAGAT ATATCTTGGTGCG-3' in the secondary PCR" should read "primer sets 5'-ATAGTCTCCGCTGTATTC-3' and 5'-GGAAG GAACGATGTATCT-3' in the primary PCR and 5'-TCCGCTGTATTCTCAGCC-3' and 5'-GCAGAGGAACCAGCATC-3' in the secondary PCR". 